\title{
Programación o planeación de actividades o recursos en la agricultura. Una revisión de literatura
}

\author{
VANESA RAMÍREZ VALENCIA \\ Diana María CÁRDENAS AgUIRRE \\ SANTIAgo Ruiz HERRERA
}

\section{Resumen}

Con el propósito de detectar las herramientas y enfoques desarrollados en la programación de recursos o la planeación de actividades en la agricultura, el presente documento muestra la revisión sistemática de literatura adelantada para tal fin, así mismo, expone los resultados y discusión de ellos. Se pretende realizar un diagnóstico del desarrollo de este sector y como está siendo impactado por diferentes herramientas de optimización, que en su mayoría han sido aplicadas en la industria. El documento muestra una introducción general, las ecuaciones de búsqueda utilizadas, las bases de datos en las que fueron aplicadas las ecuaciones de búsqueda, los resultados de la revisión sistemática de literatura y la discusión de los resultados obtenidos.

Palabras claves: Programación, Planeación, Agricultura, Actividades, Recursos, Operación.

\section{Scheduling or planning of activities or resources in agriculture. A review of the literature}

\section{Abstract}

With the purpose of detect the tools and approaches developed into the resource scheduling or an activity planning in the agriculture, the present paper shows the systematic review of literature which was done for such purpose,

1 Universidad Nacional de Colombia - Sede Manizales, Colombia.

. Autor de correspondencia: Ramírez Valencia (Vanesa): Calle 67 \# 25 - 103 Apto 301, Manizales - Caldas. Teléfono: 3214253799.

Correo electrónico: vramirezv@unal.edu.co
Historia del artículo:

Artículo recibido: 30-VIII-2017/ Aprobado: 24-IV-2018

Disponible online: 3 de agosto 2018

Discusión abierta hasta abril de 2020 
likewise, it shows the results and discussing of them. Is intended to make a diagnostic of the development into this field and how it is influenced by different optimization tools, which have been mainly applied in the industry. The paper shows a general introduction, the searching equations used, the databases where the searching equations were applied, the results of the systematic review of literature and the discussion of the results obtained.

Keywords: Scheduling, Planning, Agriculture, Activities, Resources, Operation.

\section{Programação ou planejamento de atividades ou recursos na agricultura. Uma revisão da literatura.}

\section{Resumo}

Para detectar as ferramentas e abordagens desenvolvidas na programação de recursos ou o planejamento de atividades na agricultura, este documento mostra a revisão sistemática da literatura para este propósito, bem como os resultados e discussões sobre elas. Pretende-se fazer um diagnóstico do desenvolvimento deste setor e como ele está sendo impactado por diferentes ferramentas de otimização, que foram aplicadas principalmente na indústria. 0 documento apresenta uma introdução geral, as equações de busca utilizadas, os bancos de dados em que as equações de busca foram aplicadas, os resultados da revisão sistemática da literatura e a discussão dos resultados obtidos.

Palavras-chave: Programação, Planejamento, Agricultura, Atividades, Recursos, Operação.

\section{Introducción}

Gran parte del avance industrial y la preocupación por que cada día sea más eficiente la producción de bienes o servicios, ha impulsado a desarrollar diferente herramientas de optimización que han potencializado este sector. El sector agrícola por otra parte, estuvo por un tiempo fuera del foco de investigación; a mediados de la década de los 60's comienza a tener algún interés por parte de investigadores, debido a que se detecta la importancia de ver el sector agrícola como una parte fundamental en la cadena productiva, por lo que se convierte en objeto de estudio enfocado también a la optimización de operaciones o recursos.

Dada la relevancia que tiene actualmente el sector agrícola y la importancia de impulsar esta economía, el presente documento contiene una revisión sistemática de literatura, la cual, busca detectar las herramientas y enfoques desarrollados en la programación de recursos o la planeación de actividades en la agricultura; se presenta de igual manera la metodología que se siguió, como la definición de las ecuaciones de búsqueda y las bases de datos utilizadas, también se muestran los resultados obtenidos y la discusión de los mismos.

\section{Metodología de la revisión sistemáti- ca de literatura}

La metodología que se siguió en la revisión sistemática de literatura, comienza con la definición de una ecuación de búsqueda que fue utilizada en las bases de datos WEB OF SCIENCE y SCOPUS.

La ecuación de búsqueda se definió con varias palabras que se encontraron pertinentes a partir de revisiones de literatura previas, en donde se generó una búsqueda desde el título de "labor or staff or work or crew or shift or operatio* or "human resorc*" y de "scheduling or assignment or 
modeling or planning", desde todo el documento se definieron las palabras "Agri* or farm or land or plantation or coffee or harvest", esta ecuación de búsqueda se realizó el 01 de Enero del 2017 en la base de datos WEB OF SCIENCE, con un total de 407 artículos encontrados. Adicional a esto, se definió la ecuación de búsqueda desde el título de "labor or staff or work or crew or shift or operatio* or "human resorc*" y de "scheduling or assignment or modeling or planning", desde el título, resumen y palabras claves, se definieron las palabras "Agri* or farm or land or plantation or coffee or harvest", esta ecuación de búsqueda se realizó el 29 de Marzo del 2017 en la base de datos SCOPUS, con un total de 415 artículos encontrados.

En total se encontraron 130 artículos repetidos entre una base de datos y otra, por lo que en total fueron 692 artículos, de los cuales hubo 6 artículos que no fueron posibles de encontrar, los otros 686 se analizaron desde el título, resumen y palabras claves, para poder realizar una clasificación previa; de esta clasificación se seleccionaron 54 artículos, los cuales se leyeron en su totalidad, de estos artículos se seleccionaron dos que no eran pertinentes para el presente documento, por los que se obtuvo la clasificación que se puede observar en la Tabla 1.
TABLA 1. RESULTADOS DE LA ECUACIÓN DE BÚSQUEDA. FUENTE : ELABORACIÓN PROPIA.

\begin{tabular}{c|c} 
Clasificación de artículos & Total \\
\hline Relevantes & 52 \\
\hline No relevantes & 634 \\
\hline No encontrados & 6 \\
\hline Total & 692 \\
\hline
\end{tabular}

Los artículos que se identificaron como relevantes, son los que se centraron en el tema de programación de recursos o actividades, enfocadas directamente a la agricultura, estos artículos se analizarán en el siguiente apartado. Los artículos no relevantes son los que no tienen aporte y tratan temáticas muy diferentes a las actualmente investigadas.

\section{Principales resultados obtenidos de la revisión de literatura}

De la ecuación de búsqueda definida en el apartado anterior que fue aplicada en las bases de datos WEB OF SCIENCE y SCOPUS, se identificaron 52 artículos relevantes (Tabla 1), ya que estos artículos han desarrollado soluciones que se han aplicado para mejorar la eficiencia en la programación de recursos o actividades, enfocadas directamente a la agricultura, los autores que se centran en estos temas se pueden observar en la Tabla 2 .

TABLA 2. AUTORES QUE HAN INVESTIGADO SOBRE PROGRAMACIÓN O PLANEACIÓN DE RECURSOS O ACTIVIDADES, ENFOCADAS DIRECTAMENTE A LA AGRICULTURA Y SUS PRINCIPALES OBJETIVOS.

FUENTE: ELABORACIÓN PROPIA.

\begin{tabular}{|l|l|}
\hline \multicolumn{1}{|c|}{ Autores } & \multicolumn{1}{c|}{ Objetivo } \\
\hline $\begin{array}{l}\text { Van Wy y Hatting } \\
(1964)\end{array}$ & $\begin{array}{l}\text { El cálculo de la cantidad de trabajo requerido para las empresas agrícolas, por lo general pro- } \\
\text { porciona muchos problemas; la razón principal de esto es la gran variedad de necesidades de } \\
\text { mano de obra para las granjas con diferentes combinaciones y el número limitado de normas } \\
\text { disponibles en relación con los requerimientos de trabajo de estas empresas. El primer paso en } \\
\text { la planificación de las necesidades de mano de obra de una granja, es determinar las normas } \\
\text { de trabajo físico para cada actividad agrícola. El propósito de este análisis es dar orientación } \\
\text { sobre cómo la organización agrícola puede ser planificada y obtener menos mano de obra por } \\
\text { unidad de producción. }\end{array}$ \\
\hline Stuart (1971) & $\begin{array}{l}\text { En este artículo se presta atención a la programación lineal y programación cuadrática, mien- } \\
\text { tras que hace hincapié en el uso del método de Monte Carlo, se comparan estos métodos, y se } \\
\text { analizan cada uno por separado para la aplicación en la agricultura. }\end{array}$ \\
\hline
\end{tabular}




\begin{tabular}{|c|c|}
\hline Van Elderen (1978) & $\begin{array}{l}\text { Las operaciones de programación en una granja se consideran en función de los hombres y las } \\
\text { máquinas disponibles y sobre la influencia de las condiciones meteorológicas en los materiales } \\
\text { (contenido de humedad). El modelo de simulación con la estrategia heurística se utiliza para la } \\
\text { selección de las operaciones en cada momento. El modelo de programación lineal se utiliza en } \\
\text { la cosecha de granos para demostrar la influencia de los modelos en los costes variables (horas } \\
\text { extras, las pérdidas por la no puntualidad en el secado del grano, la paja y el trigo) y la influen- } \\
\text { cia de los datos de entrada (el tiempo, atributos del material y número de horas laborables) en } \\
\text { aquello costos. }\end{array}$ \\
\hline Van Elderen (1980) & $\begin{array}{l}\text { Este estudio compara la simulación heurística, la programación lineal y la programación dinámi- } \\
\text { ca, en la toma de decisiones en las fincas de trigo, dirigido a soluciones factibles y razonables. La } \\
\text { decisión de los momentos de las operaciones, se realiza con una urgencia. La estrategia de las } \\
\text { fincas de trigo asigna una urgencia para cada combinación y al mismo tiempo busca la combi- } \\
\text { nación con la máxima urgencia. }\end{array}$ \\
\hline Wijngaard (1988) & $\begin{array}{l}\text { El problema básico que aquí se considera es la programación de los puestos de trabajo. Los } \\
\text { ejemplos incluyen la cosecha de grano, empacado de paja y arado. La programación consiste } \\
\text { en asignar los hombres y las máquinas a los puestos de trabajo y la asignación de puestos de } \\
\text { trabajo a los períodos de tiempo. El objetivo es la minimización del costo. La asignación se lle- } \\
\text { van a cabo bajo varias restricciones: el número de puestos de trabajo, los equipos y hombres } \\
\text { disponibles y las propiedades de los materiales (estos últimos están relacionados con el clima). }\end{array}$ \\
\hline $\begin{array}{l}\text { Thangavadivelu y } \\
\text { Colvin (1997) }\end{array}$ & $\begin{array}{l}\text { Desarrollar un sistema de apoyo para tomar decisiones basadas en lógica difusa (FLoDSS) para } \\
\text { ayudar a los administradores de granjas a decidir si debe o no llevar a cabo la operación de } \\
\text { labranza en un día determinado. Fue probado y evaluado, utilizando los datos de previsión me- } \\
\text { teorológica y las decisiones diarias realizada por dos encargados de la granja. Este sistema mues- } \\
\text { tra de acuerdo con la humedad del suelo si es factible o no realizar la operación de labranza. }\end{array}$ \\
\hline $\begin{array}{l}\text { Joannon et al. } \\
\text { (2005) }\end{array}$ & $\begin{array}{l}\text { Evaluar el margen de maniobra de los agricultores para introducir el arado de rastrojo y la siem- } \\
\text { bra de mostaza. se analizó la carga de trabajo y se simularon secuencias de operaciones de culti- } \\
\text { vo para un clima promedio para las } 14 \text { granjas principales ubicadas en la cuenca. Este análisis se } \\
\text { basó en encuestas realizadas con agricultores. Posteriormente se determinó el número de días } \\
\text { de trabajo disponibles para el arado de rastrojo y la siembra de mostaza. }\end{array}$ \\
\hline $\begin{array}{l}\text { Sørensen y Niel- } \\
\text { sen (2005) }\end{array}$ & $\begin{array}{l}\text { Llevar a cabo estudios agrícolas de equipos innovadores de labranza, para cuantificar los di- } \\
\text { versos insumos y recursos, incluyendo parámetros de un modelo, para los modelos de tareas } \\
\text { generalizadas. El segundo paso incluyó la modificación de un número de sistemas de máquinas } \\
\text { que reflejan los diversos métodos disponibles para llevar a cabo las operaciones necesarias para } \\
\text { la preparación del suelo, la siembra y el cuidado de las parcelas en escenarios que implican la } \\
\text { adopción integral de métodos de labranza reducida. }\end{array}$ \\
\hline $\begin{array}{l}\text { Corner y Foulds } \\
\text { (2005) }\end{array}$ & $\begin{array}{l}\text { Realizar un informe sobre el desarrollo y aplicación de un modelo de programación para un } \\
\text { escenario particular que involucra la recolección de bloques forestales. El objetivo primario del } \\
\text { estudio de caso de recolección reportado en este trabajo, es especificar la actividad de cada } \\
\text { trabajador y cada máquina, en cada período de tiempo, con el fin de minimizar la duración de la } \\
\text { recolección de todo el bloque. }\end{array}$ \\
\hline $\begin{array}{l}\text { Tiwaria y Giteb } \\
\text { (2006) }\end{array}$ & $\begin{array}{l}\text { El principal objetivo del estudio fue identificar un programa de trabajo y reposo, para un ren- } \\
\text { dimiento óptimo de las actividades, sin ningún tipo de molestia indebida (disconfort) para los } \\
\text { operadores, estos resultados se pueden utilizar para preparar directrices para el funcionamiento } \\
\text { de la cultivadora. }\end{array}$ \\
\hline
\end{tabular}




\begin{tabular}{|c|c|}
\hline $\begin{array}{l}\text { Weintraub y } \\
\text { Romero (2006) }\end{array}$ & $\begin{array}{l}\text { Analizar y evaluar el rendimiento pasado de los modelos de investigación de operaciones en } \\
\text { la agricultura, resaltar los problemas actuales, las direcciones futuras de la investigación y las } \\
\text { aplicaciones. Se centra en la planificación a escala de fincas y del sector regional, las implicacio- } \\
\text { nes ambientales, las cuestiones de riesgo e incertidumbre. En la parte forestal, se enfoca en la } \\
\text { planificación de problemas en los niveles estratégico, táctico y operacional, las cuestiones de } \\
\text { implementación, las implicaciones ambientales, así como el tratamiento de la incertidumbre y } \\
\text { múltiples objetivos. }\end{array}$ \\
\hline $\begin{array}{l}\text { Foulds y Zhao } \\
\text { (2007) }\end{array}$ & $\begin{array}{l}\text { Describir el Sistema de Soporte de Decisiones (DSS) desarrollado para la programación de las } \\
\text { operaciones de los contratistas que viajan de una granja a otra con el maíz de la cosecha. Mai- } \\
\text { zeManager está diseñado para ayudar a los programadores de maquinarias de cosecha y de } \\
\text { personal en la creación o mejora de horarios, y permite el uso de su experiencia y preferencias. }\end{array}$ \\
\hline $\begin{array}{l}\text { Tittonell et al. } \\
\text { (2007) }\end{array}$ & $\begin{array}{l}\text { Analizar los compromisos entre estrategias de asignación de mano de obra y sus recursos, en } \\
\text { los diferentes campos (clases de calidad del suelo) dentro de sus fincas en términos de lograr al- } \\
\text { tos rendimientos físicos. Garantizar la seguridad alimentaria, mejorar la eficiencia de la captura } \\
\text { de nutrientes y reducir las pérdidas de suelo en un sistema simplificado de granjas. }\end{array}$ \\
\hline $\begin{array}{l}\text { Ali, Verlinden y Van } \\
\text { Oudheusden (2008) }\end{array}$ & $\begin{array}{l}\text { Desarrollar por medio de un método automatizado, la planificación de itinerarios a un "costo } \\
\text { mínimo" que puedan ser seguidos por una o varias cosechadoras disponibles para la cosecha. El } \\
\text { objetivo general de la planificación es minimizar la distancia total recorrida por las cosechado- } \\
\text { ras para cosechar un campo de cultivo, minimizando así la duración del proceso de recolección. }\end{array}$ \\
\hline $\begin{array}{l}\text { Berruto y Busato } \\
\text { (2008) }\end{array}$ & $\begin{array}{l}\text { El propósito de este estudio fue simular la cosecha de arroz y las operaciones de transporte en } \\
\text { todo el complejo agrícola, para estudiar el impacto de la trazabilidad de campo [FT] en compa- } \\
\text { ración con la trazabilidad de Tiempo [TC] y teniendo en cuenta el coste de la operación. }\end{array}$ \\
\hline Guan et al. (2008) & $\begin{array}{l}\text { Desarrollar un flujo de trabajo en fincas, con un modelo híbrido de redes de Petri, aplicado a } \\
\text { la caña de azúcar. Este flujo incluye la programación del trabajo agrícola y la selección y asig- } \\
\text { nación de maquinaria y mano de obra para determinar las operaciones sobre el terreno, en un } \\
\text { lapso corto para la producción efectiva del cultivo. Así mismo se pretende diseñar un modelo } \\
\text { apropiado para modelar el flujo de trabajo agrícola para las granjas geográficamente dispersas. }\end{array}$ \\
\hline Ferrer et al. (2008) & $\begin{array}{l}\text { Desarrollar una herramienta práctica para programar de manera óptima las operaciones de } \\
\text { cosecha de uva, teniendo en cuenta tanto los costos operativos y la calidad de la uva. Busca un } \\
\text { modelo de optimización que equilibra los costos operativos del proceso de recolección, con los } \\
\text { efectos de pérdida de calidad de la uva. }\end{array}$ \\
\hline Bochtis et al. (2009) & $\begin{array}{l}\text { Realizar un modelo de eventos discretos para la predicción de distancias recorridas de una má- } \\
\text { quina que opera en actividades de manejo de materiales utilizando el concepto de CTF. }\end{array}$ \\
\hline $\begin{array}{l}\text { Bochtis y Oksane } \\
\text { (2009) }\end{array}$ & $\begin{array}{l}\text { Se presenta el primer intento de conectar estos dos enfoques ( El primer enfoque, utiliza méto- } \\
\text { dos de predicción y búsqueda exhaustiva para obtener los resultados de descomposición ópti- } \\
\text { ma del campo en sub-campos y la dirección de conducción óptima en cada campo. El segundo, } \\
\text { el uso de un algoritmo de optimización combinatoria heurístico para determinar la secuencia } \\
\text { óptima de la máquina a los sub-campos), a fin de proporcionar un método completo para la } \\
\text { planificación de la cobertura del área del campo, que es directamente aplicable a máquinas } \\
\text { agrícolas autónomas, así como a la próxima generación de auxiliares de navegación y de siste- } \\
\text { mas de autodirección. }\end{array}$ \\
\hline
\end{tabular}




\begin{tabular}{|c|c|}
\hline Khani et al. (2010) & $\begin{array}{l}\text { Desarrollar y verificar un modelo, con el fin de determinar la probabilidad de desarrollar las acti- } \\
\text { vidades de labranza en diferentes regiones, por diferentes características climáticas y del suelo. }\end{array}$ \\
\hline Naivinit et al. (2010) & $\begin{array}{l}\text { Construir una representación compartida de las interacciones entre la agricultura RLR, la dis- } \\
\text { ponibilidad de agua y la migración laboral. El propósito de este modelo no es estimar con pre- } \\
\text { cisión el efecto de la disponibilidad de agua sobre la migración laboral, sino investigar cómo } \\
\text { están interrelacionados durante la época de cosecha del arroz. }\end{array}$ \\
\hline $\begin{array}{l}\text { Pardo, Riravololona } \\
\text { y Munier-Jolain } \\
(2010)\end{array}$ & $\begin{array}{l}\text { El objetivo del presente estudio fue analizar las consecuencias de la adopción de los sistemas de } \\
\text { cultivo IWM (gestión integrada de malezas) ensayados de } 2000 \text { a 2006, este ensayo se enfocaba } \\
\text { principalmente en: 1) la organización del trabajo a nivel de finca y 2) la rentabilidad económica. }\end{array}$ \\
\hline Hameed et al. (2010) & $\begin{array}{l}\text { Presentar un método para la generación en tiempo real de las representaciones geométri- } \\
\text { cas de campos para la planificación operativa. La representación considera campos simples o } \\
\text { complejos tanto para los límites de los campos convexos como no convexos, donde las pistas } \\
\text { generadas pueden ser rectas o curvas. }\end{array}$ \\
\hline Banhara et al. (2010) & $\begin{array}{l}\text { Este estudio está dirigido a mejorar la operación de recolección actual y la planificación. Esto } \\
\text { permite la evaluación de diferentes escenarios, con limitaciones medioambientales y de ali- } \\
\text { mentación, aumento de la capacidad operativa, y las consecuencias espaciales de envío de } \\
\text { equipos a cosechar a ciertas distancias durante el período de evaluación. }\end{array}$ \\
\hline $\begin{array}{l}\text { Arnaout y Maatouk } \\
(2010)\end{array}$ & $\begin{array}{l}\text { Minimizar los costos de recolección de la uva, enfocado a asignar mejor los días de recolección, } \\
\text { teniendo en cuenta la optimización de la calidad del vino. }\end{array}$ \\
\hline $\begin{array}{l}\text { Da Silva, Alves y } \\
\text { Da Costa (2011) }\end{array}$ & $\begin{array}{l}\text { Este trabajo se centra en el desarrollo y uso de un modelo de simulación discreta para de- } \\
\text { terminar los turnos de trabajo de los operadores de equipos agrícolas de una planta en Sao } \\
\text { Paulo, teniendo en cuenta las necesidades de molienda, el riesgo de falta de material y el límite } \\
\text { permitido de horas de trabajo. El estudio cumplió con el objetivo de desarrollar un modelo } \\
\text { de simulación de operaciones de corte, carga y transporte de la caña de azúcar que podrían } \\
\text { utilizarse para evaluar el trabajo de vehículos y maquinaria. }\end{array}$ \\
\hline Eakin et al. (2011) & $\begin{array}{l}\text { Utilizar un proceso de Redes Analíticas para sintetizar el conocimiento experto sobre los prin- } \\
\text { cipales impulsores del cambio en los medios de subsistencia en una región, así como sobre las } \\
\text { estrategias familiares más comunes. Se desarrollará un marco de análisis de decisiones multi- } \\
\text { criterio y conocimientos expertos para estructurar sistemáticamente el proceso de evaluación } \\
\text { no solo para elucidar las relaciones de causa-efecto hipotéticas, sino también para enfatizar la } \\
\text { naturaleza sistémica del cambio socioecológico. }\end{array}$ \\
\hline $\begin{array}{l}\text { Costa, Menesatti y } \\
\text { Spinelli (2012) }\end{array}$ & $\begin{array}{l}\text { El objetivo de este estudio fue explorar el potencial de enfoques multivariados diferentes de la } \\
\text { OLS (es decir, PCR y PLS), centrándose principalmente en la regresión PLS al desarrollar mode- } \\
\text { los de rendimiento de la explotación forestal. }\end{array}$ \\
\hline $\begin{array}{l}\text { Ruiz-Torres et al. } \\
\text { (2012) }\end{array}$ & $\begin{array}{l}\text { Este documento propone un modelo para la planificación táctica de operaciones, que ayudaráa } \\
\text { a los cultivadores de flores con las decisiones de asignación de tierras y la variedad de flores } \\
\text { de tal manera, que los ingresos se maximicen. Esta planificación ayuda a adaptarse a los cam- } \\
\text { bios del mercado, tales como la fluctuación de los precios y la demanda, de las diferentes va- } \\
\text { riedades de plantas cosechadas. El problema considera las limitaciones de capacidad, la fuerza } \\
\text { de trabajo y la demanda, el rendimiento y variabilidad de los precios. }\end{array}$ \\
\hline Bochtis et al. (2013) & $\begin{array}{l}\text { (1) La formulación del problema de planificación de operaciones secuenciales de múltiples } \\
\text { campos como una tienda de flujo, con tiempos de configuración como parte del problema de } \\
\text { programación.(2) La demostración de la implementación del enfoque utilizando. (3) Evaluar } \\
\text { el impacto de la incertidumbre en la predicción de tiempos de trabajo sobre la aplicabilidad } \\
\text { del enfoque. }\end{array}$ \\
\hline
\end{tabular}




\begin{tabular}{|c|c|}
\hline $\begin{array}{l}\text { Bakhtiari et al. } \\
\text { (2013) }\end{array}$ & $\begin{array}{l}\text { Realizar planes óptimos de cobertura de campo para las operaciones de cosecha, utilizando los } \\
\text { patrones B de principio de secuencia de derrotas óptimo. }\end{array}$ \\
\hline Ooster et al. (2013) & $\begin{array}{l}\text { El objetivo fue identificar parámetros con fuerte influencia en el desempeño laboral, así como } \\
\text { el efecto de la incertidumbre en los parámetros de entrada en los indicadores claves de des- } \\
\text { empeño. Se analizó la sensibilidad diferencial y se probaron los resultados para la linealidad } \\
\text { del modelo. Los objetivos de este estudio fueron identificar: 1) los parámetros de entrada que } \\
\text { deben elegirse con cuidado para no comprometer la exactitud de la predicción del modelo, } \\
\text { así como parámetros para los cuales la especificación exacta es menos necesaria, 2) las carac- } \\
\text { terísticas del sistema de cultivo al que la demanda de mano de obra es muy sensible y que } \\
\text { podría guiar al diseñador y productor de un sistema en crecimiento a un sistema mejorado y } \\
\text { 3) Fuentes de incertidumbre sobre la capacidad del modelo para discriminar entre escenarios } \\
\text { de trabajo alternativos. }\end{array}$ \\
\hline $\begin{array}{l}\text { Alaiso, Backman y } \\
\text { Visala (2013) }\end{array}$ & $\begin{array}{l}\text { El contratista que presta servicio a empresas agrícolas, para realizar trabajos mecanizados, reci- } \\
\text { be típicamente hasta } 200 \text { pedidos individuales al año. Con las órdenes, tienen plazos variables, } \\
\text { programación óptima de trabajo de forma rápida. Un algoritmo se propone para la programa- } \\
\text { ción de tareas en este documento, así como el modelo del proceso de contratación en sí. }\end{array}$ \\
\hline $\begin{array}{l}\text { Edwards, Bochtis y } \\
\text { Søresen (2013) }\end{array}$ & $\begin{array}{l}\text { Desarrollar un algoritmo de optimización y programación dirigido a la programación de múlti- } \\
\text { ples tareas en múltiples lugares para varios vehículos. }\end{array}$ \\
\hline Baio et al. (2013) & $\begin{array}{l}\text { Desarrollar un modelo computacional mediante programación lineal basado en plataforma } \\
\text { web para seleccionar automáticamente los sistemas agrícolas mecanizados basados en el me- } \\
\text { nor costo operacional. }\end{array}$ \\
\hline Zhou et al. (2014) & $\begin{array}{l}\text { Desarrollar un método de planificación que genere un plan factible de cobertura de área, para } \\
\text { máquinas agrícolas que ejecuten operaciones no capacitadas en campos con múltiples áreas } \\
\text { y obstáculos. }\end{array}$ \\
\hline $\begin{array}{l}\text { Marques, Sousa y } \\
\text { Rönnqvistb (2014) }\end{array}$ & $\begin{array}{l}\text { Explorar el potencial del uso de técnicas de optimización en combinación con modelos de } \\
\text { simulación de eventos discretos (DES) para planificar operaciones de cosecha y logística, que } \\
\text { reconozcan la incertidumbre. }\end{array}$ \\
\hline $\begin{array}{l}\text { Beaudoin, Frayret y } \\
\text { Lebel (2014) }\end{array}$ & $\begin{array}{l}\text { Presentar un proceso de planificación táctica, basado en el enfoque genérico de modelos je- } \\
\text { rárquicos de Schneeweiss. Esta planificación busca, primero que todo, anticipar solo parte del } \\
\text { nivel operacional para evaluar la información más relevante para el tomador de decisiones tác- } \\
\text { ticas y evaluar la factibilidad operativa de un plan, y en segundo lugar, evaluar el costo mínimo } \\
\text { total de transporte de equipos en la implementación del plan táctico seleccionado. }\end{array}$ \\
\hline Wishon et al. (2015) & $\begin{array}{l}\text { Determinar el número apropiado de trabajadores para plantación y cosecha, para adquirir la } \\
\text { mano de obra necesaria con anterioridad, desarrolla planificaciones de tácticas de nivelación, } \\
\text { cosecha y adquisición de mano de obra, para determinar el número adecuado de trabajadores } \\
\mathrm{H}-2 \mathrm{~A} \text { (trabajadores externos), aplicado a frutas y hortalizas. }\end{array}$ \\
\hline Ooster et al. (2015) & $\begin{array}{l}\text { Analizar modelos basados en la gestión de mano de obra, orientada a las habilidades de los } \\
\text { trabajadores en un sistema de producción hortícola multioperaciones y multifuncionales. }\end{array}$ \\
\hline $\begin{array}{l}\text { Pathumnakul y } \\
\text { Nakrachata-Amon } \\
\text { (2015) }\end{array}$ & $\begin{array}{l}\text { Analizar las aplicaciones de la investigación de operaciones para los problemas experimen- } \\
\text { tados en la planificación de la cosecha de caña de azúcar. Los problemas que se discuten en } \\
\text { este documento incluyen el problema de enrutamiento de las cosechadoras, el problema de } \\
\text { fusión de campos y el problema de la integración de la fuerza de trabajo y la cosechadora en } \\
\text { las operaciones. }\end{array}$ \\
\hline
\end{tabular}


Desarrollar un enfoque para predecir y optimizar el rendimiento general de todas las operaciones, dado un campo seleccionado y las máquinas requeridas. Desarrollar una herramienta de planificación de la gestión para la toma de decisiones sobre decisiones operativas (por ejemplo, la dirección de conducción, la posición de recarga) y el dimensionamiento de la maquinaria (por ejemplo, el tamaño del tanque / tolva). Por ultimo desarrollar un modelo de simulación para múltiples operaciones secuenciales en una producción de papa y demostrar las capacidades de este modelo como un sistema de apoyo a la decisión para la gestión de operaciones.

Jensen et al. (2015)

Identificar las actividades no productivas en las operaciones de campo que incluyen maquinaria, se descompone el proceso de cobertura de área en acciones de conducción factibles y analizar el potencial de minimizar las actividades no productivas.

Probar un enfoque de planificación de rutas, utilizando un robot de comportamiento determi-

Bochtis et al. (2015) nístico (denominado AMS - sistema de mecanización autónomo). Esta investigación proporcionará un sistema completo de planificación de rutas para vehículos de huertos autónomos.

El objetivo de este trabajo es desarrollar una herramienta para la programación de maquinaria agrícola para la recolección de biomasa y operaciones de manipulación en una serie de cam-

Edwards et al. pos geográficamente dispersos que tenga en cuenta la preparación de campos. Desarrollar un (2015) algoritmo de programación que crea planes de trabajo de máquinas individuales, para múltiples máquinas para ejecutar múltiples operaciones consecutivas en múltiples campos, lo que explica la disponibilidad del campo para la operación especificada.

Desarrollar un enfoque algorítmico para la optimización de operaciones en campo, utilizando el caso de la fertilización líquida. El objetivo del proceso de optimización es generar una des-

Jensen, Bochtis y cripción de alto nivel de los trayectos de conducción, un plan de cobertura sujeto a las restric-

Sørensen (2015) ciones, bajo el criterio de minimizar el total de la distancia recorrida no productiva. El plan de cobertura describe en qué secuencia las pistas deben ser trabajadas, cuando se debe trabajar una pista parcial o completamente, cuando se deben rellenar y cómo debe llegar al depósito.

El objetivo de este estudio fue desarrollar una metodología que ayude a crear una logística detallada del plan de trabajo para los gestores de las fincas, mediante la definición de las estra-

Montgomery, Han y tegias utilizadas en el norte del condado de Humboldt, California, utilizando el análisis espacial Kizha (2016) para identificar las ubicaciones óptimas para la molienda centralizada y los desembarques de remolques, basados en la distribución espacial de la biomasa, las redes de carreteras existentes y las características del terreno.

Presentar un modelo para predecir el costo de las operaciones en el campo y del transporte

Sopegno et al. para múltiples cultivos. El modelo propuesto se refiere al nivel estratégico de toma de decisio(2016) nes en un sistema de producción agrícola relacionado con el diseño del sistema de mano de obra / maquinaria en relación con los tipos de cultivos seleccionados.

(1) caracterizar y obtener datos de proceso de las operaciones actuales de cosecha en planta-

Engler, Becker y

Hoffmann (2016) ciones forestales en el sur de China. (2) desarrollar un modelo de productividad de recolección basado en el rendimiento actual (consumo de tiempo, productividad y costos de operación). (3) Desarrollar modelos aplicables para mejorar las operaciones mecanizadas.

El propósito de la investigación es desarrollar y perfeccionar la teoría de la demanda de trabajo, la estimación y la mano de obra rural excedente en la industria de la siembra, y luego a

Fulin, Shengxue y proporcionar algunas referencias teóricas para la estimación científica. El modelo establecido

Xiaoming (2016) en esta investigación se puede utilizar no sólo para calcular la cantidad de demanda de trabajo actual, si no también en los diferentes momentos del futuro previsto de acuerdo al nivel de mecanización y las áreas cultivadas. 


\begin{tabular}{|l|l|}
\hline Monjezi et al. (2016) & $\begin{array}{l}\text { En este trabajo se presenta el método GERT basado en la teoría difusa para la solución de } \\
\text { programación de proyectos de producción de caña de azúcar (preservar las operaciones de } \\
\text { cosecha, y el racionamiento). }\end{array}$ \\
\hline Cunha et al. (2016) & $\begin{array}{l}\text { Evaluar la utilización de la metodología de superficie de respuesta (RSM) para verificar el efecto } \\
\text { de la velocidad de operación, la longitud media de las filas de café y la pendiente de las áreas, } \\
\text { en los parámetros de desempeño operacional en la cosecha de café súper mecanizada. }\end{array}$ \\
\hline
\end{tabular}

\section{Discusión de resultados:}

La Tabla 2 muestra los autores que, según la búsqueda realizada, han enfocado sus investigaciones en optimizar la programación o planeación de recursos en la agricultura para diferentes cultivos, con diversos métodos de solución, como se puede ver con Joannon, et al. (2005), quienes buscan generar más flexibilidad en la programación de operaciones o recursos, para que los agricultores puedan ser más asertivos, y lo basa en encuestas a ellos mismos. Autores como Sørensen y Nielsen (2005), tratan el recurso humano en operaciones agrícolas, pero combinado con maquinaria para observar cómo pueden tener mayor rendimiento ciertas actividades. De la misma manera, Guan, et al. (2008), también tratan la maquinaria junto con el recurso humano, pero con el propósito de desarrollar un flujo de trabajo en los cultivos de caña de azúcar.

Adicional a esto, Corner y Foulds (2005), exponen a partir de la especificación de cada actividad, tanto de maquinaria como de personal, la forma de minimizar la recolección de bloques forestales. Autores como, Foulds y Zhao (2007), también examinan la maquinaria de cosecha y el personal, pero con el objetivo de mejorar los horarios de trabajo; existen investigaciones en el análisis desarrollado, que se centran igualmente en el bienestar del trabajador, en donde tienen en cuenta los reposos y el programa de trabajo, para que no resulte ningún tipo de molestia indebida, involucrando las cultivadoras (Tiwaria y Giteb, 2006). Autores como Ooster, et al. (2013), se centran en el desempeño laboral. Así mismo, otros autores se enfocan en el rendimiento, la programación o planificación de tareas, desde una área más administrativa (Costa, Menesatti y Spinelli, 2012; Alaiso, Backman y Visala, 2013; Marques, Sousa y Rönnqvistb, 2014), estos enfoques se hacen indispensables en el análisis de un cultivo para genera rentabilidad en los negocio agrícolas.

Se pudo encontrar en la literatura analizada en la Tabla 2, autores como Ali, Verlinden y Van Oudheusden (2008), Berruto y Busato (2008), Bochtis, et al. (2009), Bochtis y Oksane (2009), Hameed, et al. (2010), Bakhtiari, et al. (2013) y Edwards, Bochtis y Søresen (2013); los cuales centran sus investigaciones en el recorrido de una máquina y como pueden desarrollar algún tipo de optimización o mejora en este tema en específico, lo cual toma una mayor importancia a medida que las operaciones en campo se automatizan.

Así mismo, se encuentra autores como Ferrer, et al. (2008) y Arnaout y Maatouk (2010), que centran sus estudios en el cultivo de uva, en donde no solo tienen en cuenta la optimización de las operaciones y la reducción de costos, si no también incluyen la calidad del fruto en el momento de la recolección, ya que esto afecta significativamente la calidad del vino producido; Monjezi, et al. (2016), también desarrolla su investigación en la agricultura, pero lo propone desde la programación de proyectos de producción de caña de azúcar. Bochtis, et al. (2013), desarrolla una investigación en la que se centra en la planificación de operaciones secuenciales, pero incluye en su estudio la incertidumbre de la predicción de tiempos en el trabajo, lo que genera unos resultados más asertivos y aplicables a la industria agrícola.

En la revisión actual se encontró que existen autores que involucran las condiciones del suelo en sus investigaciones (Tittonell, et al., 2007; Khani, et 
al., 2010), así como, la disponibilidad del agua (Naivinita, et al., 2010) y condiciones medioambientales o ecológicas (Banhara, et al., 2010; Eakin, et al., 2011); que al tratarse de la agricultura el objeto de estudio analizado para el actual documento, es de importancia incluir esta variables en las investigaciones y los autores anteriormente mencionado logran darle la relevancia pertinente.

Existen autores de los observados en la Tabla 2, que tratan la optimización de operaciones desde el recurso humano, como lo son Wishon, et al. (2015), que buscan encontrar el número apropiado de trabajadores para unas plantaciones; Ooster, et al. (2015), que se centran únicamente en las habilidades de los trabajadores que realizan las operaciones en la horticultura, para obtener resultados más eficientes y Da Silva, Alves y Da Costa (2011), que incorpora en su investigación, el límite de horas de trabajo permitidas para un hombre. Sopegno, et al. (2016), se interesa en los mismos temas que los autores analizados anteriormente, pero difiere al enfocarse en la toma de decisiones estratégicas en la agricultura, estos autores incluyen tanto la mano de obra, como la maquinaria en todo el desarrollo del proyecto.

Desde el punto de análisis de operaciones mecanizadas o semi mecanizadas, encontramos autores como Engler, Becker y Hoffmann (2016), que buscan, a partir de su análisis de actividades, impulsar la mecanización con el soporte del rendimiento de las actividades desarrolladas. Así mismo, Pathumnakul y Nakrachata-Amon (2015), buscan avanzar en las operaciones mecanizadas y proponen como tema innovador, la fusión de campos, para que se puedan realizar las operaciones con máquinas en pequeñas fincas. Cunha, et al. (2016), centra toda su investigación en el proceso de recolección de café mecanizado, para evaluar su desempeño operacional. Autores como Fulin, Shengxue y Xiaoming (2016), incorpora de igual manera la maquinaria agrícola en su investigación, en donde, de acuerdo al grado de mecanización de las actividades, se puede estimar la necesidad de mano de obra.
También se detectaron varios autores que se han enfocado en la producción agrícola mecanizada (Baio, et al., 2013; Beaudoin, Frayret y Lebel, 2014; Zhou, et al., 2014; Zhou, et al., 2015; Jensen, Bochtis y Sørensen, 2015; Bochtis, et al., 2015; Edwards, et al., 2015; Jensen, et al., 2015; Montgomery, Han y Kizha, 2016;), que se pueden observar a más detalle en la Tabla 2. Lo dicho hasta aquí supone que, en las actividades agrícolas se está involucrando más fuertemente la maquinaria, esto se puede dar, debido a las optimizaciones que genera el cambio de una operación manual a mecanizada, ya que, en la mayoría de ocasiones, impacta directamente en la reducción de mano de obra y tiempos de operación, que a su vez, genera reducciones en los costos totales de producción.

Una investigación que ha incluido la planificación táctica de operaciones, es la de Ruiz-Torres, et al. (2012), que ayudará a los cultivadores de flores con las decisiones de asignación de tierras y la variedad de flores, de tal manera, que los ingresos se maximicen. Esta planificación ayuda a adaptarse a los cambios del mercado, tales como la fluctuación de los precios y la demanda, de las diferentes variedades de plantas cosechadas. El problema considera las limitaciones de capacidad, fuerza de trabajo, demanda, rendimiento y variabilidad de los precios. Este proyecto, es de gran relevancia para la investigación, debido a las condiciones del cultivo y por las variables incluidas en el estudio, ya que incluyen la selección del tipo de flor de acuerdo con la demanda y precio, algo que no se ha incluido en ningún otro estudio analizado hasta el momento. Autores como Pardo, Riravololona y Munier-Jolain (2010), toman factores como las malezas, las cuales también es importante investigarlas ya que afectan de manera positiva o negativamente la rentabilidad del negocio, dependiendo de tipo de cultivo.

Para finalizar, se resaltan los trabajos de Wijngaard (1988) y Thangavadivelu y Colvin (1997), ya que fueron identificados como relevantes, pues son pioneros en aplicación de herramientas de programación de operaciones en la agricultura, con apor- 
tes como la programación de puestos de trabajo y la consideración del clima como criterio para saber si es óptimo o no realizar actividades de labranza. No se pueden dejar de un lado, los autores que también trataron este tema, como lo son, Van Wyk y Hatting (1964), Stuart (1971), Van Elderen (1978) y Van Elderen (1980). Weintraub Y Romero (2006), realizan un análisis acerca de la aplicación de la investigación de operaciones en la agricultura y su evolución, en donde se observa que ha sido altamente aplicada y ha tenido una evolución rápida en el sector, y señalan retos para futuros investigadores en el tema, al incluir variables propias del sector agrícola.

$\mathrm{Al}$ observar cada investigación detalladamente, se pudieron encontrar los siguientes resultados de relevancia: el 55,8 \% de las investigaciones, se centran solo en programación o planeación de actividades agrícolas, el 38,5 \%, se enfocaron en la combinación de programación o planeación de actividades agrícolas y los recursos que las realizan, mientras que solo un 5,7 \% de las investigaciones se centraron en los recursos que realizan las actividades. Por lo que se pudo concluir, que lo que toma más relevancia en el campo de la investigación agrícola, esta orientado a las actividades, no a los recursos con los que realizan las operaciones.

Por otra parte, los recursos que realizan las actividades agrícolas, que más se han estudiados son: la maquinaria con un $42,3 \%$, la combinación de la maquinaria y la mano de obra con un $21,2 \%$, la mano de obra con un 19,2 \% y un 17,3\% de las investigaciones no especifican los recursos o incluyen algún otro solo estudiado en ese artículo. De estos resultados se concluyó, que la maquinaria agrícola es de alta relevancia en los estudios analizados, seguido por el recurso humano que desarrolla la labor agrícola. Así mismo, se detectó que, el 50 \% de las investigaciones incluidas en este documento, aplican modelos matemáticos para darle solución a los problemas planteados o realizar las investigaciones pertinentes, y un 13,5 \%, recurrió a la simulación como herramienta de investigación.

\section{Conclusiones}

Como conclusión principal, se puede observar que la programación de operaciones o recursos en la agricultura, ha sido estudiada hace más de 50 años; se identifica como un tema de gran relevancia para varios autores, no solo por los aportes al crecimiento y desarrollo de este sector de la economía, sino por los retos que genera la aplicación de las herramientas de programación, a las condiciones particulares del sector agrícola. Así mismo, se detecta un desafío importante más allá de las condiciones de la agricultura (condiciones meteorológicas, de terrenos, etc.), como lo es, las variaciones de acuerdo al cultivo (especificaciones del producto que se cultiva), ya que, como se detectó en la discusión de literatura, cada investigación se enfoca en un cultivo, lo que genera, variables muy diversas en el campo de la agricultura, enfocadas al objeto de estudio.

Adicional a esto, como se pudo observar en varias de las investigaciones mencionadas en la Tabla 2, la programación adecuada de recursos o actividades genera un impacto en los costos de las operaciones estudiadas, de igual manera, se detectaron los siguientes factores importantes en la asignación o programación de actividades o recursos: condiciones del cultivo, recurso que realiza las actividades, forma de realizar las operaciones, condiciones meteorológicas, forma de programar y calidad del producto. Hay que mencionar, además, que todo lo analizado y concluido en el actual documento genera un visión clara de los cultivos que se están estudiando y las herramientas de programación y planeación que se están teniendo en cuenta para este campo de estudio, lo que genera una base de investigaciones enfocadas al desarrollo agrícola y que pueden ser de utilidad como insumo para llevar acabo investigaciones enfocadas a la optimización de actividades o recursos en la agricultura. 


\section{Referencias}

Alaiso, S.; Backman, J.; Visala, A. (2013). Ant Colony Optimization for Scheduling

of Agricultural Contracting Work. IFAC Proceedings Volumes, 46(18), pp. 133-137. [Online] Disponible en: $\quad$ http://dx.doi.org/10.3182/20130828-2SF-3019.00041 [Consultado 30 de Marzo de 2017]

Ali, O.; Verlinden, B.; Van Oudheusden, D. (2008). Infield logistics planning for crop-harvesting operations. Engineering Optimization, 41(2), pp. 183-197. [Online] Disponible en: http://dx.doi. org/10.1080/03052150802406540 [Consultado 31 de Marzo de 2017]

Arnaout, J. P. M.; Maatouk, M. (2010). Optimization of quality and operational costs through improved scheduling of harvest operations. International Transactions in Operational Research, 17(5), pp. 595-605. [Online] Disponible en: http://dx.doi. org/10.1111/j.1475-3995.2009.00740.x [Consultado 30 de Marzo de 2017]

Baio, F. H.; Rodrigues, A. D.; Santos, G. S. D.; Silva, S. P. D. (2013). Mathematical modeling to select mechanized agricultural systems by the lowest operational cost. Engenharia Agrícola, 33(2), pp. 402-410. [Online] Disponible en: http://dx.doi.org/10.1590/ S0100-69162013000200018 [Consultado 30 de Marzo de 2017]

Bakhtiari, A.; Navid, H.; Mehri, J.; Berruto, R.; Bochtis, D. D. (2013). Operations planning for agricultural harvesters using ant colony optimization. Spanish Journal of Agricultural Research, 11(3), pp. 652-660. [Online] Disponible en: http://dx.doi.org/10.5424/ sjar/2013113-3865 [Consultado 30 de Marzo de 2017]

Banhara, J. R.; Rodriguez, L. C. E.; Seixas, F.; Moreira, J. M. M.; da Silva, L. M. S.; Nobre, S. R.; Cogswell, A. (2010). Optimized harvest scheduling in eucalyptus plantations under operational, spatial and climatic constraints. Scientia Forestalis, 38(85), pp. 85-95. [Online] Disponible en: https://www.scopus.com/ inward/record [Consultado 29 de Marzo de 2017]

Beaudoin, D., Frayret, J., y Lebel, L. (2014) Hierarchical forest management with anticipation: an application to tactical-operational planning integration. Canadian Journal of Forest Research, 38(8), pp. 2198-221.
[Online] Disponible en: https://doi.org/10.1139/ X08-055 [Consultado 29 de Marzo de 2017]

Berruto, R.; Busato, P. (2008). System approach to biomass harvest operations: simulation modeling and linear programming for logistic design. InASABE Annual International Meeting, Rhode Island, Paper (No. 084565). [Online] Disponible en: https:// www.scopus.com/inward/record [Consultado 29 de Marzo de 2017]

Bochtis, D. D.; Dogoulis, P.; Busato, P.; Sørensen, C. G.; Berruto, R.; Gemtos, T. (2013). A flow-shop problem formulation of biomass handling operations scheduling. Computers and electronics in agriculture, 91, pp. 49-56. [Online] Disponible en: https://doi. org/10.1016/j.compag.2012.11.015 [Consultado 30 de Marzo de 2017]

Bochtis, D.; Griepentrog, H. W.; Vougioukas, S.; Busato, P.; Berruto, R.; y Zhou, K. (2015). Route planning for orchard operations. Computers and Electronics in Agriculture, 113, pp. 51-60. [Online] Disponible en: https://doi.org/10.1016/j.compag.2014.12.024 [Consultado 30 de Marzo de 2017]

Bochtis, D. D.; Sørensen, C. G.; Jørgensen, R. N.; Green, O. (2009). Modelling of material handling operations using controlled traffic. biosystems engineering, 103(4), pp. 397-408. [Online] Disponible en: https://doi.org/10.1016/j.biosystemseng.2009.02.006 [Consultado 30 de Marzo de 2017]

Bochtis, D. D.; Oksanen, T. (2009). Combined coverage path planning for field operations. In Proc. Joint International Agricultural Conference, JIAC, pp. 521527. [Online] Disponible en: https://www.scopus. com/inward/record [Consultado 29 de Marzo de 2017]

Corner, J. L.; Foulds, L. R. (2005). Scheduling the Harvesting Operations of a Forest Block: A Case Study. AsiaPacific Journal of Operational Research, 22(03), pp. 377-390. [Online] Disponible en: https://doi. org/10.1142/S0217595905000674 [Consultado 29 de Marzo de 2017]

Costa, C.; Menesatti, P.; Spinelli, R. (2012). Performance modelling in forest operations through partial least square regression. Silva Fennica, 46(2), pp. 241-252. [Online] Disponible en: https://doi.org/10.14214/ sf.57 [Consultado 30 de Marzo de 2017] 
Cunha, J. P.; Silva, F. M. D.; Andrade, E. T. D.; Carvalho, L. C. (2016). Modeling of operational performance parameters applied in mechanized harvest of coffee. Revista Brasileira de Engenharia Agrícola e Ambiental, 20(10), pp. 946-952. [Online] Disponible en: http://dx.doi.org/10.1590/1807-1929/agriambi.v20n10p946-952 [Consultado 30 de Marzo de 2017]

Da Silva, J.E.A.R.; Alves, M.R.P.A.; Da Costa, M.A.B. (2011). Planejamento de turnos de trabalho: uma abordagem no setor sucroalcooleiro com uso de simulação discreta. Gestão \& Produção, 18(1), pp. 73-90. [Online] Disponible en: http://dx.doi.org/10.1590/ S0104-530X2011000100006 [Consultado 31 de Marzo de 2017]

Eakin, H.; Bojórquez-Tapia, L. A.; Diaz, R. M.; Castellanos, E.; Haggar, J. (2011). Adaptive capacity and socialenvironmental change: theoretical and operational modeling of smallholder coffee systems response in Mesoamerican Pacific Rim. Environmental management, 47(3), pp. 352-367. [Online] Disponible en: https://doi.org/10.1007/s00267-010-9603-2 [Consultado 31 de Marzo de 2017]

Edwards, G.; Bochtis, D.; Søresen, C. G. (2013). Multimachine coordination: Scheduling operations based on readiness criteria and using a modified tabu search algorithm. IFAC Proceedings Volumes, 46(18), pp. 191-195. [Online] Disponible en: $\quad$ http://dx.doi.org/10.3182/20130828-2SF-3019.00023 [Consultado 30 de Marzo de 2017]

Edwards, G.; Sørensen, C. G.; Bochtis, D. D.; Munkholm, L. J. (2015). Optimised schedules for sequential agricultural operations using a Tabu Search method. Computers and Electronics in Agriculture, 117, pp. 102-113. [Online] Disponible en: https://doi. org/10.1016/j.compag.2015.07.007] [Consultado 29 de Marzo de 2017]

Engler, B.; Becker, G.; Hoffmann, S. (2016). Process mechanization models for improved Eucalyptus plantation management in Southern China based on the analysis of currently applied semi-mechanized harvesting operations. Biomass and Bioenergy, 87, pp. 96-106. [Online] Disponible en: https://doi. org/10.1016/j.biombioe.2016.02.021 [Consultado 29 de Marzo de 2017]

Ferrer, J. C.; Mac Cawley, A.; Maturana, S.; Toloza, S.; Vera, J. (2008). An optimization approach for scheduling wine grape harvest operations.International Journal of Production Economics, 112(2), pp. 985-999. [Online] Disponible en: https://doi.org/10.1016/j. ijpe.2007.05.020 [Consultado 30 de Marzo de 2017]

Foulds, L. R.; Zhao, X. D. (2007). A Decision Support System for sustainable maize harvesting operations scheduling. International Journal of Business Information Systems, 2(4), pp. 372-391. [Online] Disponible en: http://dx.doi.org/10.1504/IJBIS.2007.012541 [Consultado 30 de Marzo de 2017]

Fulin, W.; Shengxue, Z.; Xiaoming, F. (2016). Improved estimation model and empirical analysis of relationship between agricultural mechanization level and labor demand. International Journal of Agricultural and Biological Engineering, 9(2), pp. 48. [Online] Disponible en: 10.3965/j.ijabe.20160902.2188 [Consultado 30 de Marzo de 2017]

Guan, S.; Nakamura, M.; Shikanai, T.; Okazaki, T. (2008). Hybrid Petri nets modeling for farm work flow. Computers and electronics in agriculture, 62(2), pp. 149-158. [Online] Disponible en: https://doi. org/10.1016/j.compag.2007.12.006 [Consultado 30 de Marzo de 2017]

Hameed, I. A.; Bochtis, D. D.; Sørensen, C. G.; Nøremark, M. (2010). Automated generation of guidance lines for operational field planning. Biosystems Engineering, 107(4), pp. 294-306. [Online] Disponible en: https://doi.org/10.1016/j.biosystemseng.2010.09.001 [Consultado 30 de Marzo de 2017]

Jensen, M. F.; Bochtis, D.; Sørensen, C. G. (2015). Coverage planning for capacitated field operations, part II: Optimisation. Biosystems Engineering, 139, pp. 149-164. [Online] Disponible en: https://doi. org/10.1016/j.biosystemseng.2015.07.002 [Consultado 30 de Marzo de 2017]

Jensen, M. F.; Nørremark, M.; Busato, P.; Sørensen, C. G.; Bochtis, D. (2015). Coverage planning for capacitated field operations, Part I: Task decomposition. Biosystems Engineering, 139, pp. 136-148. [Online] Disponible en: https://doi.org/10.1016/j.biosystemseng.2015.07.003 [Consultado 30 de Marzo de 2017]

Joannon, A.; Papy, F.; Martin, P.; Souchère, V. (2005). Planning work constraints within farms to reduce runoff at catchment level. Agriculture, ecosystems y environment, 111(1), pp. 13-20. [Online] Disponible en: 
https://doi.org/10.1016/j.agee.2005.04.021 [Consultado 30 de Marzo de 2017]

Khani, M.; Keyhani, A.; Parsinejad, M.; Alimardani, R. (2010). Verification and sensitivity analysis of a model for determination of probability of a working day for tillage. International Agrophysics, 25(1), pp. 27-35. [Online] Disponible en: http://www.old. international-agrophysics.org/ [Consultado 29 de Marzo de 2017]

Marques, A. F.; de Sousa, J. P.; Rönnqvist, M. (2014). Combining optimization and simulation tools for shortterm planning of forest operations.Scandinavian Journal of Forest Research, 29(sup1), pp. 166-177. [Online] Disponible en: http://dx.doi.org/10.1080 /02827581.2013.856937 [Consultado 30 de Marzo de 2017]

Monjezi, N.; Sheikhdavoodi, M. J.; Zakidizaji, H.; Marzban, A.; Shomeili, M. (2016). Operations scheduling of sugarcane production using fuzzy GERT method (part II: preserve operations, harvesting and ratooning). Agricultural Engineering International: CIGR Journal, 18(3), pp. 343-349. [Online] Disponible en: http://www.cigrjournal.org/ [Consultado 31 de Marzo de 2017]

Montgomery, T. D.; Han, H. S.; Kizha, A. R. (2016). Modeling work plan logistics for centralized biomass recovery operations in mountainous terrain. Biomass and Bioenergy, 85, pp. 262-270. [Online] Disponible en: http://dx.doi.org/10.1016/j.biombioe.2015.11.023 [Consultado 31 de Marzo de 2017]

Naivinit, W.; Le Page, C.; Trébuil, G.; Gajaseni, N. (2010). Participatory agent-based modeling and simulation of rice production and labor migrations in Northeast Thailand. Environmental Modelling y Software, 25(11), pp. 1345-1358. [Online] Disponible en: https://doi.org/10.1016/j.envsoft.2010.01.012 [Consultado 30 de Marzo de 2017]

Ooster, A.; Bontsema, J.; van Henten, E. J.; Hemming, S. (2013). Sensitivity analysis of a stochastic discrete event simulation model of harvest operations in a static rose cultivation system. Biosystems engineering, 116(4), pp. 457-469. [Online] Disponible en: https://doi.org/10.1016/j.biosystemseng.2013.10.009 [Consultado 30 de Marzo de 2017]

Ooster, A.; Bontsema, J.; van Henten, E. J.; Hemming, S. (2015). Model-based analysis of skill oriented labour management in a multi-operations and multi-worker static cut rose cultivation system. Biosystems Engineering, 135, pp. 87-102. [Online] Disponible en: https://doi.org/10.1016/j.biosystemseng.2015.04.014 [Consultado 30 de Marzo de 2017]

Pardo, G.; Riravololona, M.; Munier-Jolain, N. M. (2010). Using a farming system model to evaluate cropping system prototypes: Are labour constraints and economic performances hampering the adoption of Integrated Weed Management?. European Journal of Agronomy, 33(1), pp. 24-32. [Online] Disponible en: https://doi.org/10.1016/j.eja.2010.02.003 [Consultado 30 de Marzo de 2017]

Pathumnakul, S.; Nakrachata-Amon, T. (2015). The Applications of Operations Research in Harvest Planning: A Case Study of the Sugarcane Industry in Thailand. Journal of the Management Association of Japan, 65(4E), pp. 328-333. [Online] Disponible en: https://www.scopus.com/inward/record [Consultado 30 de Marzo de 2017]

Ruiz-Torres, A. J., Villalobos, J. R., Salvador-Jijon, M., y Alomoto, N. (2012). Planning models for floriculture operations. International Journal of Applied Management Science, 4(2), pp. 148-164. [Online] Disponible en: http://dx.doi.org/10.1504/ IJAMS.2012.046206 [Consultado 30 de Marzo de 2017]

Sopegno, A.; Busato, P.; Berruto, R.; Romanelli, T. L. (2016). A cost prediction model for machine operation in multi-field production systems. Scientia Agricola, 73(5), pp. 397-405. [Online] Disponible en: http://dx.doi.org/10.1590/0103-9016-2015-0304 [Consultado 31 de Marzo de 2017]

Sørensen, C. G.; Nielsen, V. (2005). Operational analyses and model comparison of machinery systems for reduced tillage. Biosystems engineering, 92(2), pp. 143-155. [Online] Disponible en: https://doi. org/10.1016/j.biosystemseng.2005.06.014 [Consultado 30 de Marzo de 2017]

Stuart, 0. (1971). The utilisation of operations research techniques in the planning of agricultural undertakings. Agrekon, 10(4), pp. 12-15. [Online] Disponible en: http://dx.doi.org/10.1080/03031853.1971.95 23861 [Consultado 30 de Marzo de 2017]

Thangavadivelu, S.; Colvin, T. S. (1997) Fuzzy-LogicBased Decision Support System for Scheduling 
Tillage Operations. Engineering Applications of Artificial Intelligence, 10 (5), pp. 463-472. [Online] Disponible en: http://dx.doi.org/10.1016/S09521976(97)00023-7 [Consultado 31 de Marzo de 2017]

Tittonell, P.; Van Wijk, M. T.; Rufino, M. C.; Vrugt, J. A.; Giller, K. E. (2007). Analysing trade-offs in resource and labour allocation by smallholder farmers using inverse modelling techniques: a case-study from Kakamega district, western Kenya. Agricultural Systems, 95(1), pp. 76-95. [Online] Disponible en: https://doi.org/10.1016/j.agsy.2007.04.002 [Consultado 29 de Marzo de 2017].

Tiwari, P. S.; Gite, L. P. (2006). Evaluation of work-rest schedules during operation of a rotary power tiller. International Journal of Industrial Ergonomics, 36(3), pp. 203-210. [Online] Disponible en: https:// doi.org/10.1016/j.ergon.2005.11.001 [Consultado 30 de Marzo de 2017]

Van Wyk, S. P.; Hattingh, H. S. (1964). Labour Planning of a Diversified Farm on the Transvaal Highveld. Agrekon, 3(1), pp. 20-31. [Online] Disponible en: http://dx.doi.org/10.1080/03031853.1964.95 24453 [Consultado 30 de Marzo de 2017]

Van Elderen, E. (1978). Scheduling farm operations. European Journal of Operational Research, 4(1), pp. 19-23. [Online] Disponible en: https://doi. org/10.1016/0377-2217(80)90035-1 [Consultado 30 de Marzo de 2017]

Van Elderen, E. (1980). Models and techniques for scheduling farm operations: a comparison. Agricultural systems, 5(1), pp. 1-17. [Online] Disponible en: http:// dx.doi.org/10.1016/0308-521X(80)90016-5 [Consultado 30 de Marzo de 2017]

Weintraub, A.; Romero, C. (2006). Operations research models and the management of agricultural and forestry resources: a review and comparison. Interfaces, 36(5), pp. 446-457. [Online] Disponible en: 10.1287/inte.1060.0222 [Consultado 30 de Marzo de 2017]

Wijngaard, P. J. (1988). A heuristic for scheduling problems, especially for scheduling farm operations. European journal of operational research, 37(1), pp. 127-135. [Online] Disponible en: http://dx.doi. org/10.1016/0377-2217(88)90287-1 [Consultado 31 de Marzo de 2017]
Wishon, C.; Villalobos, J. R.; Mason, N.; Flores, H.; Lujan, G. (2015). Use of MIP for planning temporary immigrant farm labor force. International Journal of Production Economics, 170, pp. 25-33. [Online] Disponible en: https://doi.org/10.1016/j. ijpe.2015.09.004 [Consultado 31 de Marzo de 2017]

Zhou, K.; Jensen, A. L.; Bochtis, D. D.; Sørensen, C. G. (2015). Simulation model for the sequential in-field machinery operations in a potato production system. Computers and Electronics in Agriculture, 116, pp. 173-186. [Online] Disponible en: https://doi. org/10.1016/j.compag.2015.06.018 [Consultado 29 de Marzo de 2017]

Zhou, K.; Jensen, A. L.; Sørensen, C. G.; Busato, P.; Bothtis, D. D. (2014). Agricultural operations planning in fields with multiple obstacle areas.Computers and Electronics in Agriculture, 109, pp. 12-22. [Online] Disponible en: https://doi.org/10.1016/j.compag.2014.08.013 [Consultado 31 de Marzo de 2017]

\section{PARA CITAR ESTE ARTÍCULO / TO REFERENCE THIS ARTICLE / PARA CITAR ESTE ARTIGO /}

Ramírez Valencia, V.; Cárdenas Aguirre, D.M.; Ruiz Herrera, S. (2018). Programación o planeación de actividades o recursos en la agricultura. Una revisión de literatura. Revista EIA, 15(30), julio-diciembre, pp. 73-87. [Online]. Disponible en: https://doi.org/10.24050/reia.v15i30.1151 